\title{
Breaking The Monotony: Cobalt and Maleimide as a New Entrant to the Catellani Reaction
}

Tanmayee Nanda, ${ }^{\text {a }}$ Shyam Kumar Banjare, ${ }^{\text {a }}$ Supreeth Prasad, ${ }^{\mathrm{b}}$ Pragati Biswal, ${ }^{\mathrm{a}}$ Lokesh Gupta, ${ }^{\mathrm{a}}$ Bedadyuti Vedvyas Pati, ${ }^{a}$ Smruti Ranjan Mohanty, ${ }^{a}$ Dean J. Tantillo, ${ }^{* b}$ Ponneri C. Ravikumar ${ }^{* a}$

${ }^{a}$ School of Chemical Sciences, National Institute of Science Education and Research (NISER) Bhubaneswar, HBNI, Odisha 752050

${ }^{b}$ Department of Chemistry, University of California - Davis, Davis, CA 95616, USA

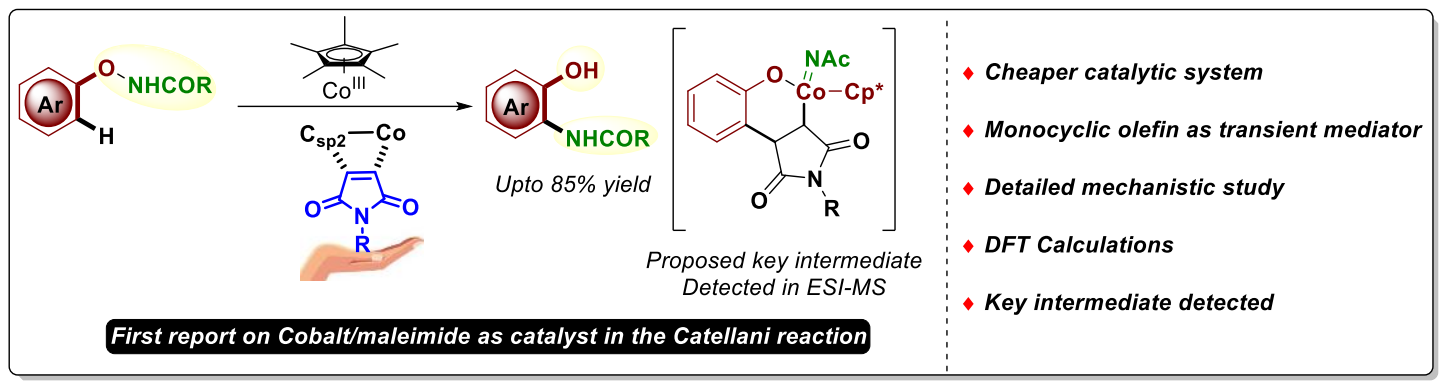

\begin{abstract}
A catalytic system was discovered for the intramolecular C-H amidation of N-phenoxy acetamide derivatives. For the first time, a cobalt catalyst was employed for the Catellani reaction. Additionally, a monocyclic olefin, maleimide, was used as a transient mediator in place of bicyclic norbornenes. Maleimide promotes a Co(III) intermediate to undergo oxidative addition into the $\mathrm{O}-\mathrm{N}$ bond to form a $\mathrm{Co}(\mathrm{V})$ nitrene species and subsequently directs nitrene addition to the ortho position. A plausible mechanism for the transformation is proposed, supported by experimental and density functional theory (DFT) computational studies. Further, the synthetic utility of this methodology was demonstrated via the orthoamidation of estrone.
\end{abstract}

\section{INTRODUCTION}

Since the dawn of the $21^{\text {st }}$ century, transition metal-catalyzed $\mathrm{C}-\mathrm{H}$ bond activation has been employed as a key strategy for the synthesis of biologically active scaffolds, functional organic materials, and pharmaceuticals. ${ }^{1}$ Due to the ubiquitous nature of $\mathrm{C}-\mathrm{H}$ bonds in organic molecules, selective functionalization of $\mathrm{C}-\mathrm{H}$ bonds remains a challenging task. However, directing groups have enabled solutions to this problem by allowing metal to activate $\mathrm{C}-\mathrm{H}$ bonds proximal to directing groups. ${ }^{2}$ Nonetheless, the nature of the substrate and coupling partner also control the threshold for the C-H bond activation process. ${ }^{3}$

Among the various transformations involving $\mathrm{C}-\mathrm{H}$ bond activation, the Catellani reaction is one of the earliest examples and occupies a unique space in organometallic chemistry. The Catellani reaction was first discovered by Marta Catellani in 1997. This reaction uses a palladium catalyst and norbornene (NBE) as the transient directing group/mediator for the synthesis of ortho bi-functional aromatic molecules (Figure 1a). ${ }^{4}$ This Pd/NBE combination has been declared as a winning combination after thousands of successful attempts (Figure 1b). ${ }^{5}$ Bicyclic olefins have been the gold standard for driving Catellani reactions for the last 24 years - primarily limited to NBE and its derivatives. Pioneering research groups like those of $\mathrm{Yu}^{6}{ }^{6}$ Glorius, $^{7} \mathrm{Ding}^{8}{ }^{8}$ and Dong ${ }^{9}$ have used modified NBEs, which exhibited high reactivity and better selectivity than NBE1 (Figure 1c). However, until now, the scope of the olefin mediator has not been expanded beyond bicyclic bridged NBEs. In addition, only noble metal palladium has been employed in the Catellani reaction, barring a couple of reports with rhodium (Figure 1d). This landscape provides an excellent opportunity to explore the reactivity of earth-abundant, eco-friendly and less expensive first-row (a) Schematic represenetation of the Catellani reaction ${ }^{4}$

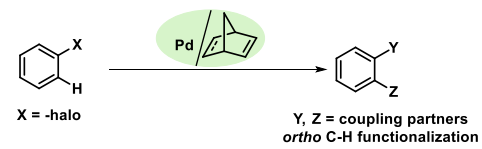

(b) Palladium/NBE catalyzed C-H functionalisation ${ }^{5}$

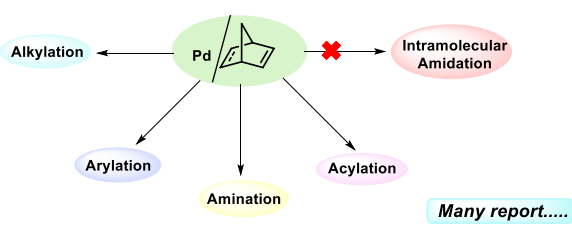

(c) Various examples of bridged bicyclic NBE and modified NBE vs maleimide.

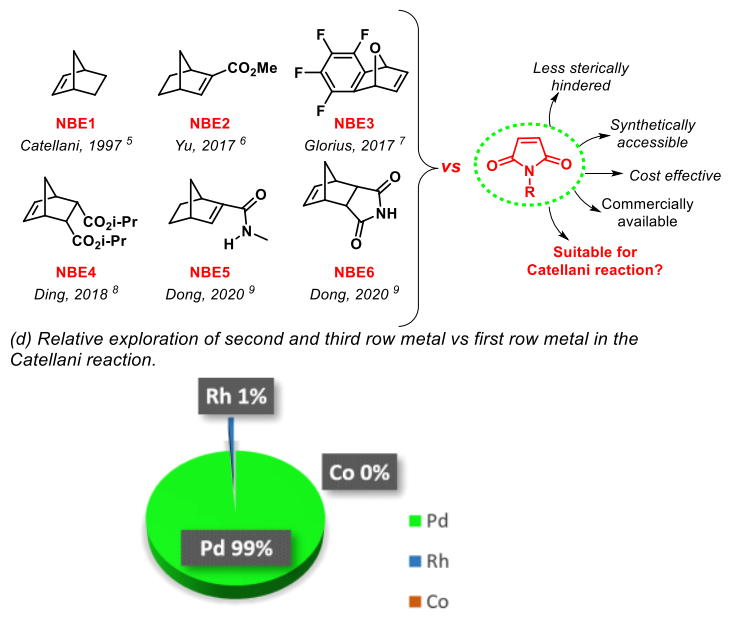

Figure 1. (a) and (b) Palladium/NBE catalyzed Catellani reaction (c) Reactivity comparison between NBE and maleimide (d) Transition metal explored in the Catellani reaction. 
transition metal such as cobalt for Catellani reactions (Figure 1d).

In 2017, the Glorius group reported an elegant Catellani approach for $o$-amidation of phenols, using a rhodium catalyst and a new oxanorbornene (NBE3) as the bicyclic olefin mediator. ${ }^{7}$ The key feature of the NBE3 structure is its rigidity, which precludes $\beta$-hydride elimination and facilitates ortho functionalization (Figure $2 \mathrm{a}$ ). ${ }^{9,10}$ Protons $\mathrm{H}_{\mathrm{a}}$ and $\mathrm{H}_{\mathrm{b}}$ do not undergo $\beta$-hydride elimination due to a lack of the requisite syn-periplanar arrangement and geometric constraints (c.f., Bredt's rule). ${ }^{11}$

To date, the potential utility of a monocyclic olefin/maleimide as a transient mediator has not been recognized by the scientific community. Therefore, we intended to perform the intramolecular $\mathrm{C}-\mathrm{H}$ amidation reaction of phenol in the presence of cationic $\mathrm{Cp}^{*} \mathrm{Co}$ as catalyst and maleimide as transient mediator. In this report, we successfully demonstrate $o$-amidation of phenols using this combination of reagents (Figure 2b).

Thus, this is the first time the Catellani reaction is found to be successful with first-row metal cobalt. Moreover, this is also the first time a monocyclic olefin has been used as a Catellani mediator. The protocol has been established with an oxidizing auto cleavable directing group that also serves as an amidating agent and facilitates the formation of a $\mathrm{C}-\mathrm{N}$ bond. The salient features of the developed protocol are: (i) intramolecular $\mathrm{C}\left(\mathrm{sp}^{2}\right)-\mathrm{H}$ amidation, (ii) a less expensive catalytic system, (iii) use of a cobalt/monocyclic olefin combination, (iv) oxidative addition of $\mathrm{Co}$ (III) to $\mathrm{O}-\mathrm{N}$ bond forming a $\mathrm{Co}(\mathrm{V})$ nitrene species, $(\mathrm{v})$ detection of a key intermediate in ESI-MS, (vi) support for the proposed mechanism through DFT calculation, and (vii) application of the method to latestage diversification of a natural product.

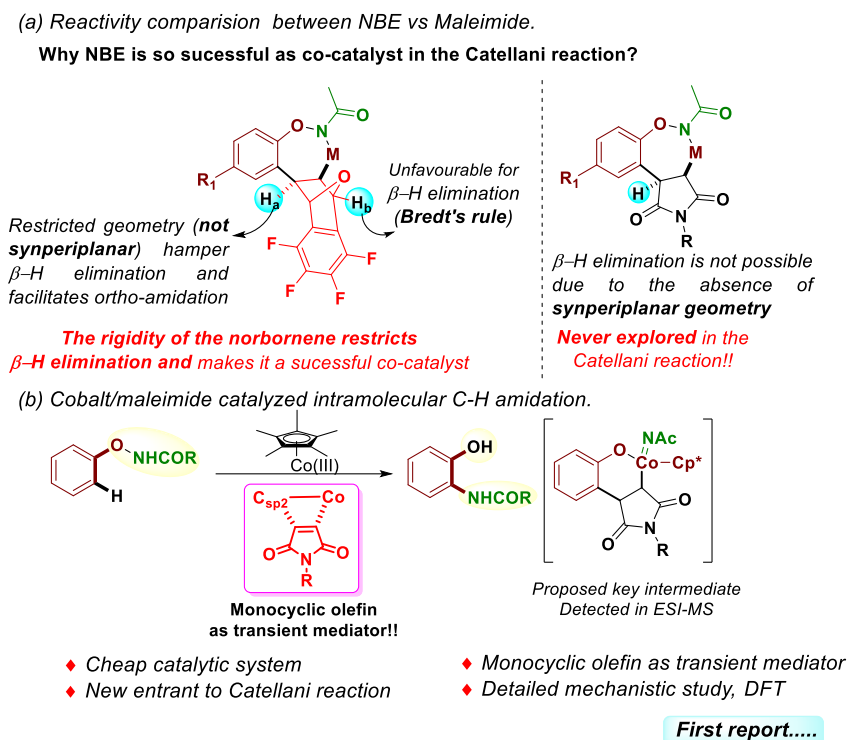

Figure 2. (a) Reactivity comparison between NBE and maleimide (b) first time reported the new catalytic system for Catellani reaction consisting of first-row Co metal and maleimide as transient mediator.

\section{RESULTS AND DISCUSSION}

We began our investigation by choosing $N$-( $p$-tolyloxy)acetamide $\mathbf{2} \mathbf{b}$ as the model substrate. A stoichiometric amount of $\mathrm{N}$-phenyl maleimide $\mathbf{1 f}$ was used as the olefin mediator (Table 1). After several sets of optimizations, $10 \mathrm{~mol} \%$ of cationic $\mathrm{Cp}^{*} \mathrm{Co}$ complex, $50 \mathrm{~mol} \%$ of $\mathrm{CsOAc}$, and $50 \mathrm{~mol} \%$ of $\mathrm{K}_{3} \mathrm{PO}_{4}$ in the presence of $0.2 \mathrm{M}$ of TFE solvent at $60{ }^{\circ} \mathrm{C}$ was found to deliver the desired $o$-amidation product $\mathbf{3 b}$ in $70 \%$ yield (Table 1, entry 1). Instead of a cationic cobalt complex, when we employed $\mathrm{Cp}^{*} \mathrm{CoCOI}_{2}$ reduced product yields were observed (Table 1, entries 2-3). Product yield decreased to $53 \%$ when catalyst loading was reduced to $5 \mathrm{~mol} \%$ (Table 1, entry 4). A lower yield was observed upon lowering the amount of maleimide from 1 equivalent to $20 \mathrm{~mol} \%$ and 50 mol \% (Table 1, entries 5-6). Changing the acetate base to $\mathrm{NaOAc}$ or KOAc did not result in much variation in the product yield (Table 1, entries 7-8). However, CsOPiv was not efficient for the transformation; it gave only $50 \%$ yield of desired product $\mathbf{3 b}$ (Table 1, entry 9). Varying the temperature from $60{ }^{\circ} \mathrm{C}$ to $40{ }^{\circ} \mathrm{C}$ or rt resulted in lower yields (Table 1, entries 10-11). Furthermore, the reaction without CsOAc and $\mathrm{K}_{3} \mathrm{PO}_{4}$ led to decreased product yield (Table 1, entries 12-13). Control experiments indicated that $\mathrm{Cp}^{*} \mathrm{Co}$ catalyst and maleimide were indispensable for the transformation (Table 1, entries 14-15). The migration of the amide group to one of the olefin termini was the most common pathway that has been observed in transition metal-catalyzed $\mathrm{C}-\mathrm{H}$ activation of phenoxy acetamide. ${ }^{12}$ However, during optimization, the hydroarylated maleimide product was either not observed or observed only in trace amounts.

\section{Table 1. Optimization of Reaction Conditions ${ }^{a}$}

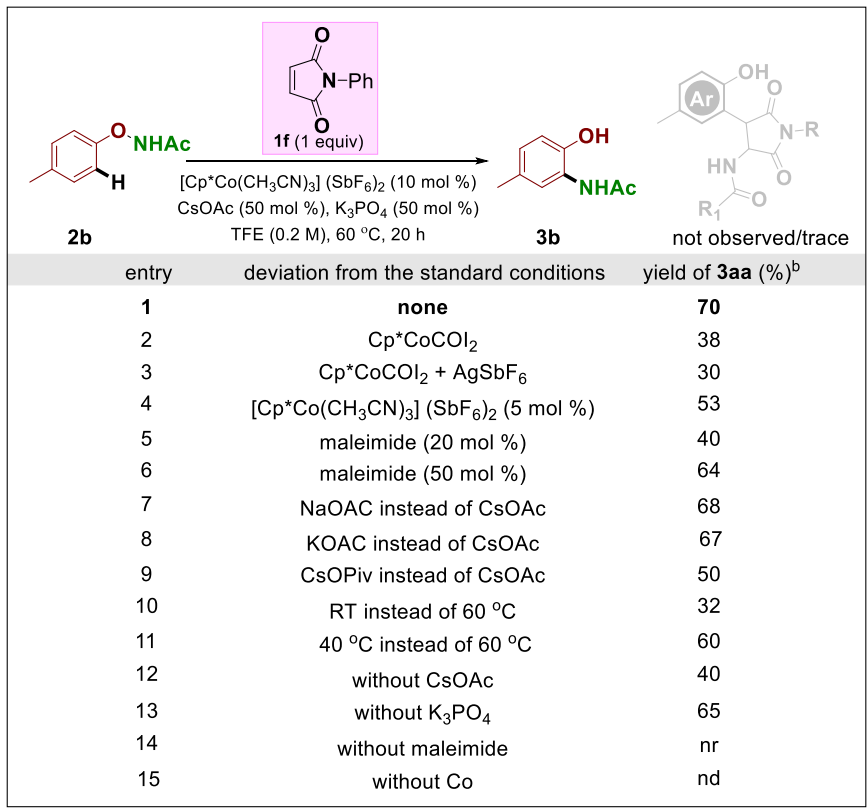

aConditions: $\mathbf{1 f}$ ( 1 equiv.), $2 \mathbf{b}$ ( 1 equiv.), [Cp* $\left.\mathrm{Co}\left(\mathrm{CH}_{3} \mathrm{CN}\right)_{3}\right]$ $\left(\mathrm{SbF}_{6}\right)_{2}(10 \mathrm{~mol} \%), \mathrm{CsOAc}(50 \mathrm{~mol} \%), \mathrm{K}_{3} \mathrm{PO}_{4}(50 \mathrm{~mol} \%), \mathrm{TFE}$ $(0.2 \mathrm{M})$, temperature $\left(60^{\circ} \mathrm{C}\right)$. bNMR yield (trimethoxybenzene was taken as internal standard for crude NMR).

We also examined an array of maleimide derivatives for cobalt catalyzed $\mathrm{C}\left(\mathrm{sp}^{2}\right)-\mathrm{H}$ amidation (Table 2). Simple maleimide 1a showed poor reactivity, providing only $30 \%$ yield of the desired product. Enhancement in the reaction yield was observed when $N$-Me $\mathbf{1 b}$ and $N$-Et 1c maleimide were employed as co-catalysts. In addition, $N$-propyl maleimide $\mathbf{1 d}$ resulted in $75 \%$ yield and $N$-benzyl maleimide 1e gave $82 \%$ 
yield of the product. As $\mathrm{N}$-Ph maleimide 1 f resulted in $70 \%$ yield of the desired product, various electron-rich and electron-poor $\mathrm{N}$-phenyl substituted maleimides were explored. The variation in substituents on the phenyl ring did not lead to much difference in reactivity, resulting in $71 \%-65 \%$ yield of the desired product (Table 2, 1g-1j). 4-Halo substituted $N$ phenyl maleimide was also tested under standard conditions, which resulted in good yield (Table 2, 1k-11). However, $N$ pentafluorophenyl maleimide decreased the product yield to $55 \%$, showing that dramatic electron deficiency on $\mathrm{N}$-substitution negatively affects the reaction.

Table 2. Investigation of Monocyclic Maleimides in the Cp*Co Catalyzed C(sp2)-H o-Amidationa

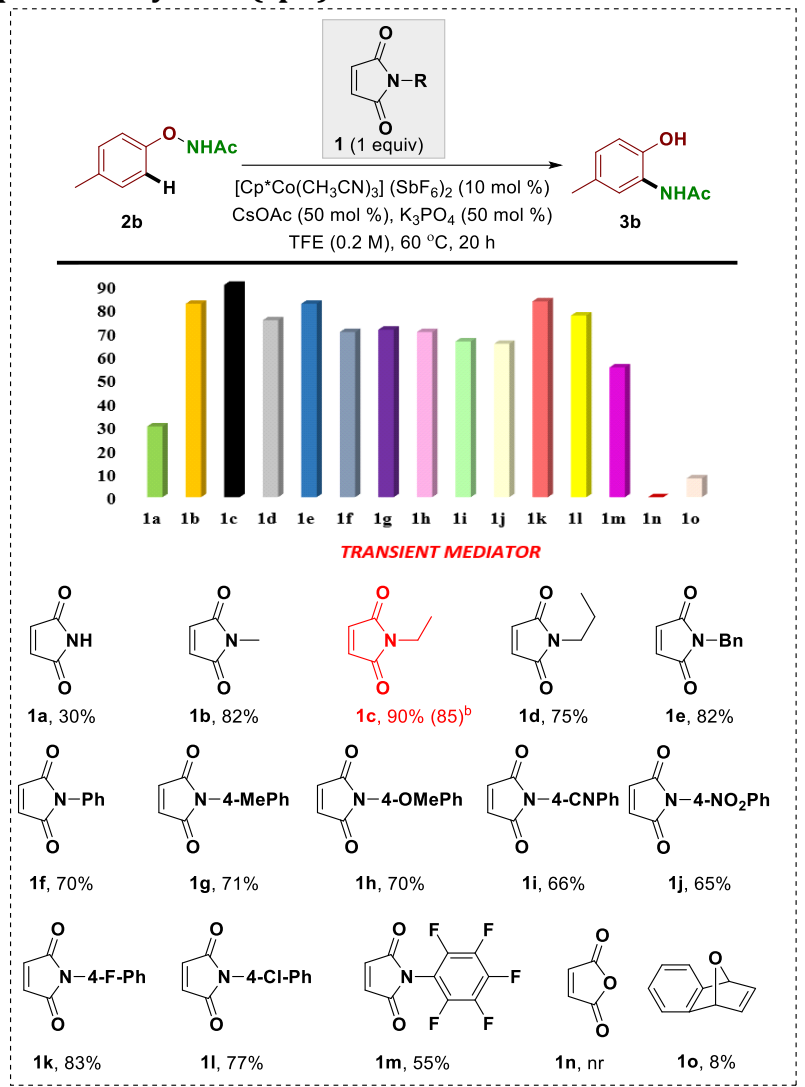

aConditions: 1 ( 1 equiv.), 2 b ( 1 equiv.), [Cp* $\left.\mathrm{Co}\left(\mathrm{CH}_{3} \mathrm{CN}\right)_{3}\right]$ $\left(\mathrm{SbF}_{6}\right)_{2}(10 \mathrm{~mol} \%), \mathrm{CsOAc}(50 \mathrm{~mol} \%), \mathrm{K}_{3} \mathrm{PO}_{4}(50 \mathrm{~mol} \%), \mathrm{TFE}$ $(0.2 \mathrm{M})$, temperature $\left(60^{\circ} \mathrm{C}\right) .{ }^{1} \mathrm{H}$ NMR yield is shown (trimethoxybenzene was taken as an internal standard for crude NMR). bResult in parentheses is the isolated yield.

The oxygen equivalent of maleimide, phthalimide 1n was also tested, however it did not to show any reactivity. The most commonly used oxanorbornene $\mathbf{1 0}$ also was employed as transient mediator and 8\% product yield was observed. This observation implies that the conditions developed for the above transformation are potentially general and cobalt/NBE combinations could serve as potential catalytic systems for other Catellani reactions in the future. In summary, $\mathrm{N}$-Et maleimide was found to be the optimal transient mediator, delivering an $85 \%$ isolated yield of the desired product.

With the optimized conditions in hand, the substrate scope was studied (Scheme 1). Unsubstituted phenoxy acetamide underwent intramolecular ortho-C-H amidation under the standard conditions to deliver $65 \%$ yield of the desired product 3a. As, $p$-methyl phenoxyacetamide $\mathbf{2} \mathbf{b}$ was found to deliver $85 \%$ yield of product during optimization, various $\mathrm{N}$ substituents were tested. Amide directing group-containing alkyl chains up to four carbons in length were found compatible, delivering $77 \%-82 \%$ yields of the desired products (Scheme 1, 3 $\mathbf{b}_{1}-\mathbf{3} \mathbf{b}_{3}$ ). In addition, branched alkyl substitution also led to good yields (Scheme 1, 3 $\mathbf{b}_{4}$ ). However, further increases in bulkiness on the directing group resulted in only a trace amount of the product $\mathbf{3} \mathbf{b}_{5}$, which can be attributed to steric repulsion during the electrophilic attack (see SI for more information).

\section{Scheme 1. Substrate Scope. ${ }^{\mathrm{a}}$}

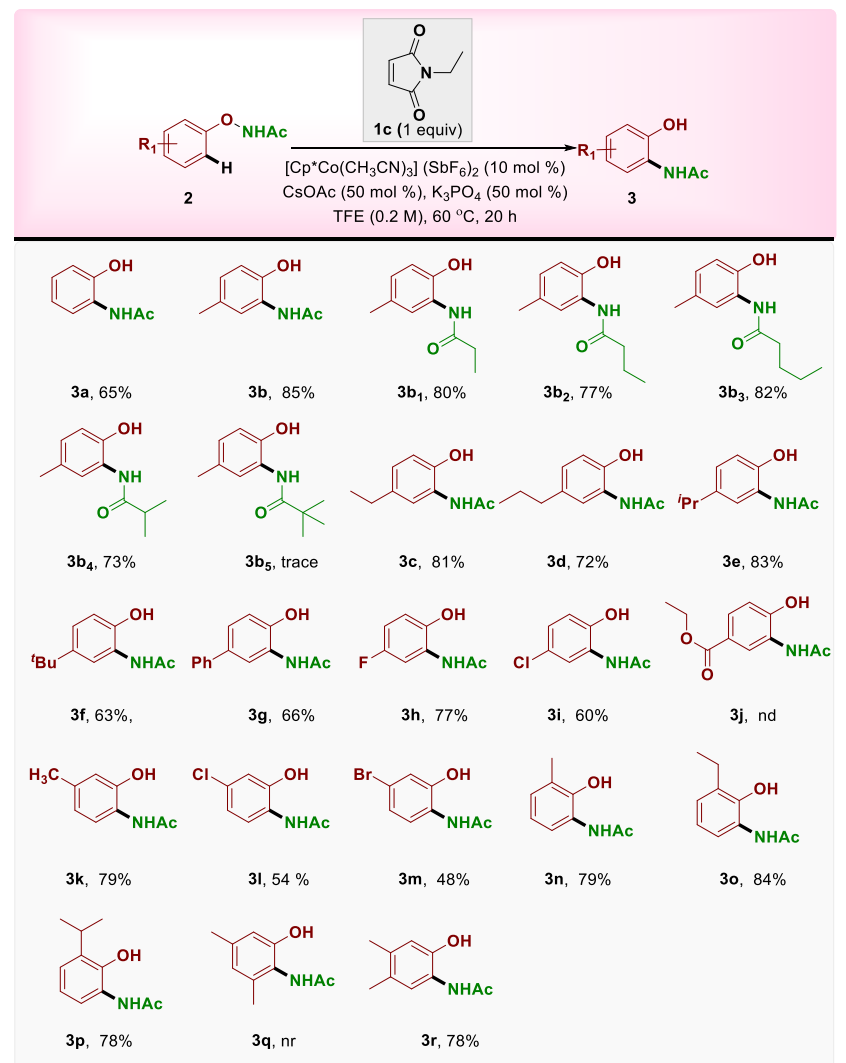

aConditions: 1 ( 1 equiv.), 2 b (1 equiv.), [Cp*Co $\left.\left(\mathrm{CH}_{3} \mathrm{CN}\right)_{3}\right]$ $\left(\mathrm{SbF}_{6}\right)_{2}(10 \mathrm{~mol} \%), \mathrm{CsOAc}(50 \mathrm{~mol} \%), \mathrm{K}_{3} \mathrm{PO}_{4}(50 \mathrm{~mol} \%), \mathrm{TFE}$ $(0.2 \mathrm{M})$, temperature $\left(60^{\circ} \mathrm{C}\right)$.

Further, the effectiveness of the standard conditions was tested with other $p$-substituted phenoxy acetamides. Alkyl (linear and branched), aryl, and halo substituted phenoxy acetamides were successfully converted to their respective products in good to very good yields (Scheme 1, 3c-3i). However, phenoxy acetamides containing electron-withdrawing groups did not result in the desired product, instead delivering carbo-amidated product $\mathbf{3}$ j* in $86 \%$ yield (see Supporting Information). This finding was supported by the DFT study, which indicates a high energy barrier for the electrophilic attack of nitrogen to the aryl ortho C-center (vide infra). Furthermore, meta substituted substrates worked well, delivering the desired products with excellent selectivity (Scheme 1, 3k-3m). Ortho-substituted substrates gave their respective products in very high yields, perhaps due to a steric-induced rate enhancement of the olefin extrusion step (Scheme 1, 3n- 
3p). However, increase in the steric effects ortho to the reacting center ( $\mathrm{C}-\mathrm{H}$ bond) shuts down reactivity. Thus, no product was detected in the case of 2q. Interestingly, meta-para disubstituted substrate $2 \mathbf{r}$ gave the desired product $\mathbf{3 r}$ in $78 \%$ yield, with the amide group transferred to the less sterically hindered site.

\section{Mechanistic Studies}

Mechanistic studies have been conducted in pursuit of insights into the details of the reaction (Scheme 2). When a cross-over experiment was performed by reacting $2 \mathbf{b}_{1}$ and 2e together, we observed products $\mathbf{3} \mathbf{b}_{\mathbf{1}}$ and $\mathbf{3 e}$ exclusively; this observation indicates that the ortho-amidation proceeded via an intramolecular pathway (Scheme 2a). The effect of electronics in the reaction rate has been tested and substrate with electron-donating substituent working better than electron withdrawing substituent (Scheme 2b). An ESIMS study led to detection of a mass peak that corresponds to the masss of cobalt-nitrenoid intermediate IV (Scheme 2c). The addition of TEMPO and BHT as radical scavengers does not impact the product yield, suggesting a non-radical pathway (Scheme 2d).

\section{Scheme 2. Mechanistic studies}

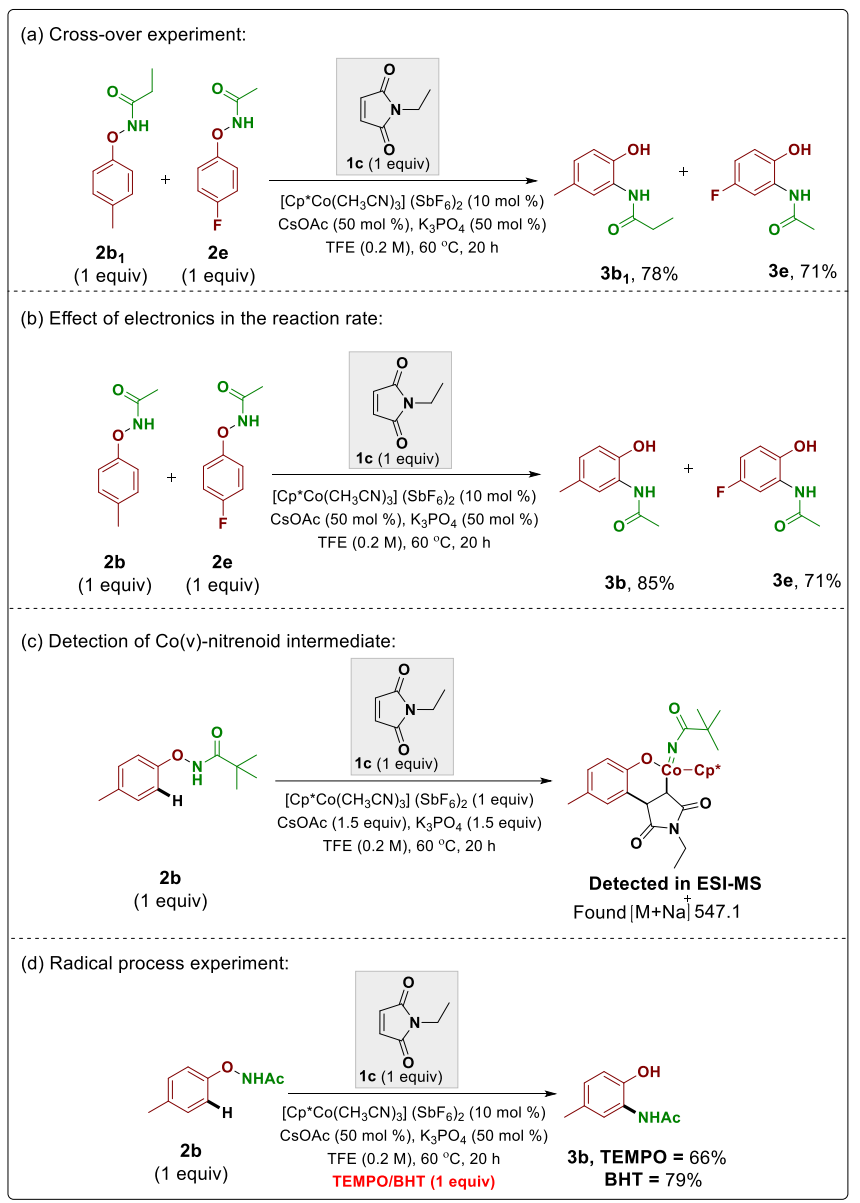

To gain additional insight into the reaction mechanism, a theoretical investigation was undertaken. DFT calculations at the M06/Def2-TZVP/SMD(TFE)//B3LYP-D3(BJ)/Def2-SVP level ${ }^{13}$ were used to examine the cascade reaction of $2 \mathbf{b}$ mediated by $\mathbf{1 b}$ as a model reaction. As the cationic catalyst $\left[\mathrm{Cp}^{*} \mathrm{Co}(\mathrm{OAc})\right]$ was proposed to be generated in the presence of CsOAc and $\mathrm{K}_{3} \mathrm{PO}_{4}$, this compound was selected as the starting point of the reaction. We also considered that a triplet state might be possible for the Co ${ }^{\text {III }}$ system, thus we performed supplementary calculations for several key structures; those calculations reveal that the energies of the triplet species are much higher than those of the corresponding singlet state species (see Supporting Information).

The mechanism considered consists of five main steps: C$\mathrm{H}$ activation, olefin insertion, oxidative addition, electrophilic addition, and olefin elimination; the complete free energy profile is shown in Figure 3. The preferred pathway for ortho $\mathrm{C}-\mathrm{H}$ bond cleavage is predicted to involve a concerted metalation deprotonation (CMD) process, ${ }^{14}$ converting intermediate 1 through the six-membered transition state structure (TSS) TS1 (Figure 3) to afford intermediate $\mathbf{2}$ (after dissociation of acetic acid), a five-membered cobaltacycle. During this process, the $\mathrm{C}-\mathrm{H}$ bond cleavage and $\mathrm{Co}-\mathrm{C}$ bond formation occur synchronously. Similar six-membered ring TSSs for CMD processes have been reported for $\mathrm{Pd}$ and $\mathrm{Ir}$ catalyzed C-H activations. ${ }^{15}$ Alternative mechanisms were considered $-\sigma$ bond metathesis, oxidative addition and electrophilic substitution - but our attempts to obtain the corresponding intermediates and/or TSSs failed. The overall free energy barrier for the CMD process is predicted to be $18.9 \mathrm{kcal} \mathrm{mol}^{-1}$, as shown in Figure 3, a readily surmountable barrier under the reaction conditions. The overall $\mathbf{0} \rightarrow \mathbf{2}$ process is predicted to be endergonic by approximately $9 \mathrm{kcal} \mathrm{mol}^{-1}$.

Once acetic acid has dissociated, the maleimide mediator can bind to $\mathbf{2}$ to form complex $\mathbf{3}$. Although complexation is predicted to be endergonic, olefin insertion via the fourmembered ring TSS TS2 (Figure 3) to form the seven-membered cobaltacycle 4 is predicted to be exergonic and rapid (Figure 1). A lower energy conformer of the seven-membered cobaltacycle, 5, then can undergo oxidative addition of Co into the $\mathrm{O}-\mathrm{N}$ bond, with a predicted barrier of $17.2 \mathrm{kcal} \mathrm{mol}^{-}$ ${ }^{1}$, through the three-membered TSS TS3 (Figure 3), forming what is formally a $\mathrm{Co}(\mathrm{V})$ nitrene species $(\mathbf{6})$.

Post $\mathrm{O}-\mathrm{N}$ cleavage, nitrene addition to the ortho carbon is predicted to be associated with a barrier of $24.3 \mathrm{kcal} \mathrm{mol}^{-1}$ (via TS4). While this is the largest barrier encountered along the reaction pathway, it is low enough to be overcome under the experimental reaction conditions. This step leads to the polycyclic dearomatized intermediate $\mathbf{7}$, which can rapidly lose maleimide via TS5 (Figure 3) to generate complex 8 (from which the final product is released by protodemetallation).

To assess the changes in aromatic character along the $6 \rightarrow$ TS4 $\rightarrow \mathbf{7} \rightarrow$ TS5 $\rightarrow \mathbf{8}$ pathway, ${ }^{16}$ we computed NICS $_{z z}$ values 1 $\AA$ Above the center of the benzene moiety (more negative values are generally associated with greater aromaticity): -25.6, $-4.7,-4.7,-8.3$, and -13.7 respectively. As expected, aromatic character is predicted to be greatest for $\mathbf{6}$ and $\mathbf{8}$, but it appears that some aromatic character is lost overall in this process, perhaps due to the presence of a second $\pi$-donating substituent attached to the benzene ring in $\mathbf{8}$. We alternatively considered that this is a direct consequence of the MillsNixon effect, ${ }^{17}$ where the aromatic benzene moiety may be slightly perturbed by the fusion of a 5-membered ring. However, there is not much bond alternation present in either 6 (1.39 ̊̊ to $1.41 \AA ̊)$ or 8 (1.38 ̊̊ to $1.42 \AA$ ). 


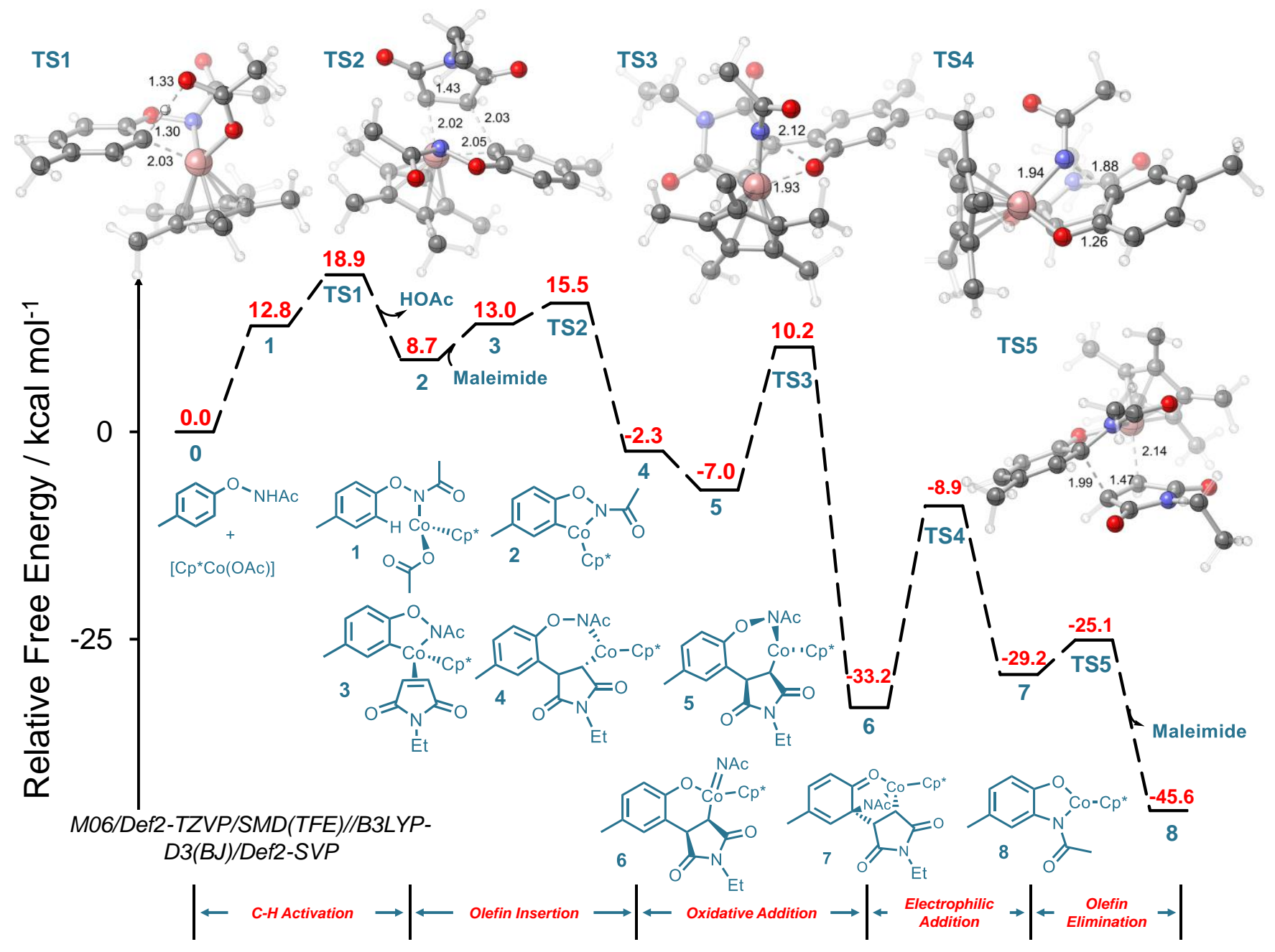

Figure 3. Proposed mechanism and optimized geometries of transition states structures (TSSs) with key nuclear distances of the $\left[\mathrm{Cp}{ }^{*} \mathrm{CoOAc}\right]$-catalyzed cascade reaction of $\mathrm{N}$-(4-methylphenoxy) acetamide mediated with $\mathrm{N}$-ethylmaleimide. The bond distances are in angstroms $(\AA)$. Color code: $\mathrm{C}$ grey, $\mathrm{H}$ white, Co pink, $\mathrm{O}$ red, $\mathrm{N}$ blue.

\section{Scheme 4. Proposed Mechanism}

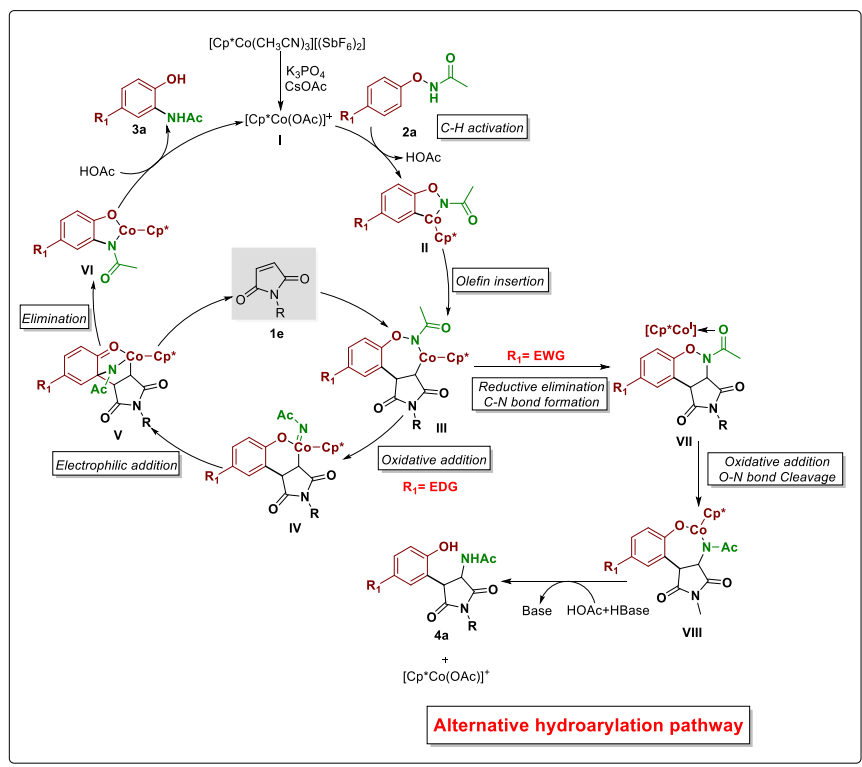

\section{Proposed Mechanism}

Based on our experimental and computational studies along with literature precedent,7,18 a plausible catalytic cycle is proposed as shown in Scheme 4. Active cationic cobalt complex I generated in situ in the presence of $\mathrm{CsOAc}$ and $\mathrm{K}_{3} \mathrm{PO}_{4}$. The cobaltacycle intermediate II is generated via a C-H activation step. Olefin insertion leads to 7-membered cobaltacycle III. $\beta$-H elimination from intermediate III has been restricted due to the absence of a syn-periplanar arrangement of the $\beta-\mathrm{H}$ and metal. Thus, the rigidity of maleimide allows intermediate III to persists and undergo oxidative addition of $\mathrm{Co}(\mathrm{III})$ to the $\mathrm{O}-\mathrm{N}$ bond, leading to the formation of $\mathrm{Co}(\mathrm{V})$ nitrene intermediate IV. At this stage, electrophilic addition of nitrogen to the ortho $\mathrm{C}-\mathrm{H}$ bond generates a dearomatized spirocyclic intermediate $\mathrm{V}$ and reducing $\mathrm{Co}(\mathrm{V})$ to $\mathrm{Co}(\mathrm{III})$. Rearomatization and steric repulsion favors the release of maleimide, resulting in intermediate VI. Further, protonation by acetic acid gives rise to product $\mathbf{3}$ and regenerates the active cationic cobalt catalyst for the next cycle. 


\section{Synthetic Utility}

The synthetic applicability of the developed protocol was demonstrated (Scheme 3). Owing to the wide availability of phenol motifs in valuable drugs and pharmaceuticals, ${ }^{19}$ achieving site-selective amidation has significant potential utility. In this context, estrone $\mathbf{4}$ was subjected to our standard conditions (Scheme 3a), and the catalytic system worked efficiently, giving $85 \%$ yield of the desired product 5 (which is superior than rhodium/NBE condition). ${ }^{7}$ Further, the reproducibility of the reaction has been checked at $1 \mathrm{mmol}$ scale and found $75 \%$ product yield (Scheme $3 \mathrm{~b}$ ). Benzoxazole core is an important pharmacophore in drug discovery. The product ortho-acetamido phenol 3a subjected to palladium catalyzed condition ${ }^{20}$ in the presence of aryl iodide delivered benzoxazole derivative 6 in $76 \%$ yield (Scheme 3c).

\section{Scheme 3. Diversification of Natural Product and Appli-} cations

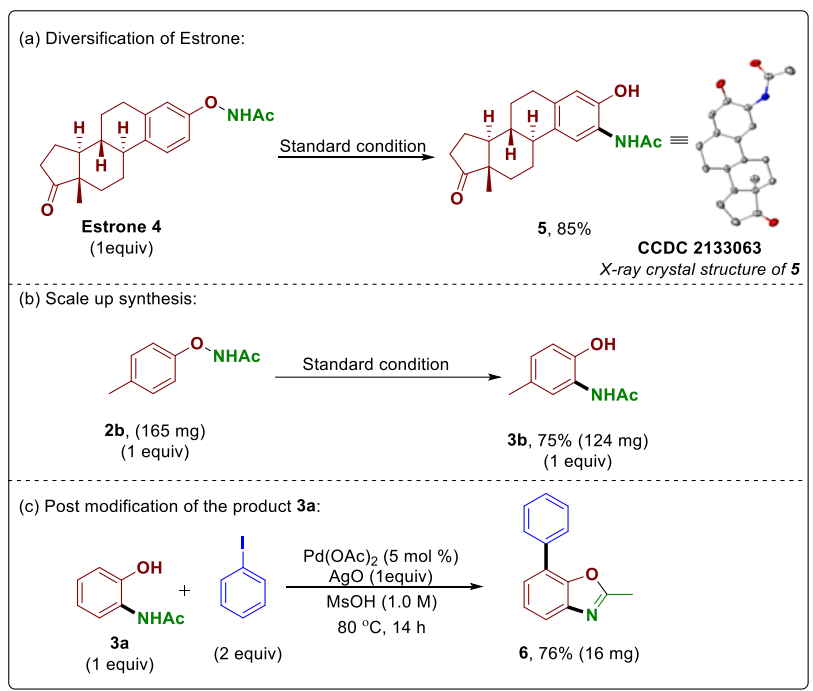

\section{CONCLUSION}

The Catellani reaction occupies a unique space in enabling numerous valuable organic transformations. In the last 24 years, Pd/NBE has been considered as a gold standard for conducting the Catellani reaction. However, in this report we disclose a cobalt catalyzed Catellani reaction, which employed a monocyclic olefin as the transient mediator, for the first time. This catalytic system is compatible with a wide range of phenoxy acetamide derivatives, delivering the desired $o$-amidated products in good yields. The synthetic utility of the developed protocol has been demonstrated on estrone derivatives, and many additional applications can be envisioned.

\section{ASSOCIATED CONTENT}

Supporting Information. This material is available free of charge via the Internet at http://pubs.acs.org.

Mechanistic studies, control experiments, and computational data, NMR spectra $\left({ }^{1} \mathrm{H},{ }^{13} \mathrm{C}\right.$, and $\left.{ }^{19} \mathrm{~F}\right)$ of $\mathbf{3 a}-\mathbf{3 r}, \mathbf{5}$, $\mathbf{6}$ and X-ray crystallography data of $\mathbf{5}$ (PDF) FAIR data, including the primary NMR FID files, for compounds 3a $-3 \mathbf{r}, 5$, and 6 (ZIP).

\section{ACCESSION CODES}

CCDC 2133063 contains the supplementary crystallographic data for this paper. These data can be obtained free of charge via www.ccdc.cam.ac.uk/data_request/cif, or by emailing data_request@ccdc.cam.ac.uk, or by contacting The Cambridge Crystallographic Data Centre, 12 Union Road, Cambridge CB2 1EZ, UK; fax: +44 1223336033.

\section{AUTHOR INFORMATION}

\section{Corresponding Authors}

Ponneri C. Ravikumar - School of Chemical Sciences, National Institute of Science Education and Research (NISER), Bhubaneswar, HBNI 752050, Odisha, India; orcid.org/ 0000-0002-5264-820X; Email: pcr@niser.ac.in

Dean J. Tantillo - Department of Chemistry, University of California-Davis, Davis, California 95616, United States; orcid.org/0000-0002-2992-8844; E-mail: djtantillo@ucdavis.edu

\section{Authors}

Tanmayee Nanda - National Institute of Science Education and Research (NISER), HBNI, Bhubaneswar 752050, India; orcid.org/0000-0001-6875-689X

Shyam Kumar Banjare - National Institute of Science Education and Research (NISER), HBNI, Bhubaneswar 752050, India; orcid.org/0000-0001-5777-7281

Supreeth Prasad - Department of Chemistry, University of California-Davis, Davis, California 95616, United States; orcid.org/0000-0003-3496-4388; E-mail: suprasad@ucdavis.edu

Pragati Biswal - National Institute of Science Education and Research (NISER), HBNI, Bhubaneswar 752050, India; orcid.org/0000-0003-4703-1923

Lokesh Gupta - School of Chemical Sciences, National Institute of Science Education and Research (NISER), HBNI, Bhubaneswar 752050, Odisha, India.

Bedadyuti Vedvyas Pati - National Institute of Science Education and Research (NISER), HBNI, Bhubaneswar 752050, India; orcid.org/0000-0002-4874-8558

Smruti Ranjan Mohanty - National Institute of Science Education and Research (NISER), HBNI, Bhubaneswar 752050, India; orcid.org/0000-0002-1218-7269

Notes

The authors declare no competing financial interest. 


\section{ACKNOWLEDGMENT}

We are thankful to NISER, Department of Atomic Energy (DAE), Council for Scientific and Industrial Research (CSIR), New Delhi (Grant 02(0256)/16/EMR II), and Science and Engineering Research Board (SERB), New Delhi (CRG/2021/007153) for the financial support. T.N, S.K.B, and P.B thank DAE NISER, L.K.G thanks SERB, S.R.M, and B.V.P thank DST INSPIRE, for research fellowship. DJT and SP acknowledge computational support from the US National Science Foundation and the XSEDE program. We are thankful to Professor Deepak Chopra, IISER Bhopal and Ms. Kasturi Sahoo NISER Bhubaneswar, for solving single crystal X-ray and Ms. Sagarika Mishra, IIT Bhubaneswar, for needful help.

\section{References}

1. (a) Lyons, T. W.; Sanford, M. S. Palladium-Catalyzed Ligand Directed $\mathrm{C}-\mathrm{H}$ Functionalization Reactions. Chem. Rev. 2010, 110, 1147-1169. (b) Gutekunst, W. R.; Baran, P. S. C-H Functionalization Logic in Total Synthesis. Chem. Soc. Rev. 2011, 40,1976-1991. (c) Hartwig, J. F. Evolution of $\mathrm{C}-\mathrm{H}$ Bond Functionalization from Methane to Methodology. J. Am. Chem. Soc. 2016, 138, 2-24. (d) Gensch, T.; Hopkinson, M. N.; Glorius, F.; Wencel-Delord, J. Mild Metal-Catalyzed $\mathrm{C}-\mathrm{H}$ Activation: Examples and Concepts. Chem. Soc. Rev. 2016, 45, 2900-2936. (e) Rej, S.; Ano, Y.; Chatani, N. Bidentate Directing Groups: An Efficient Tool in $\mathrm{C}-\mathrm{H}$ Bond Functionalization Chemistry for the Expedient Construction of $\mathrm{C}-\mathrm{C}$ Bonds. Chem. Rev. 2020, 120, 1788-1887. (f) Lam, N. Y. S.; Wu, K.; Yu, J.-Q. Advancing the Logic of Chemical Synthesis: C-H Activation as Strategic and Tactical Disconnections for C-C Bond Construction. Angew. Chem., Int. Ed. 2021, 60, 15767-15790.

2. (a) Chen, F.-J.; Zhao, S.; Hu, F.; Chen, K.; Zhang, Q.; Zhang, S.-Q.; Shi, B.-F. Pd(II)-Catalyzed Alkoxylation of Unactivated $\mathrm{C}(\mathrm{sp} 3)-\mathrm{H}$ and $\mathrm{C}(\mathrm{sp} 2)-\mathrm{H}$ bonds Using a Removable Directing Group: Efficient Synthesis of Alkyl Ethers. Chem. Sci. 2013, 4, 4187- 4192. (b) Sambiagio, C.; Schönbauer, D.; Blieck, R.; Dao-Huy, T.; Pototschnig, G.; Schaaf, P.; Wiesinger, T.; Zia, M. F.; Wencel-Delord, J.; Besset, T.; et al. A Comprehensive Overview of Directing Groups Applied in Metal-Catalysed C-H Functionalisation Chemistry. Chem. Soc. Rev. 2018, 47, 6603-6743. (c) Biswal, P.; Pati, B. V.; Chebolu, R.; Ghosh, A.; Ravikumar, P. C. Hydroxylamine-O -Sulfonic Acid (HOSA) as a RedoxNeutral Directing Group: Rhodium Catalyzed, Additive Free, One-Pot Synthesis of Isoquinolines from Arylketones. Eur. J. Org. Chem. 2020, 2020, 1006-1014.

3. (a) Crabtree, R. H.; Lei, A. Introduction: $\mathrm{CH}$ Activation. Chem. Rev. 2017, 117, 8481-8482. (b) Rej, S.; Das, A.; Chatani, N. Strategic evolution in transition metal-catalyzed directed $\mathrm{C}-\mathrm{H}$ bond activation and future directions. Coord. Chem. Rev. 2021, 431, 213683.

4. (a) Catellani, M.; Frignani, F.; Rangoni, A. A Complex Catalytic Cycle Leading to a Regioselective Synthesis of o,o'Disubstituted Vinylaromatics. Angew. Chem., Int. Ed. Engl. 1997, 36, 119-122. (b) Catellani, M.; Cugini, F. A Catalytic Process Based on Sequential ortho-Alkylation and Vinylation of ortho-Alkylaryl Iodides via Palladacycles. Tetrahedron 1999, 55, 6595-6602.

5. (a) Della Ca', N.; Fontana, M.; Motti, E.; Catellani, M. Pd/ Norbornene: A Winning Combination for Selective Aromatic Functionalization via $\mathrm{C}-\mathrm{H}$ Bond Activation. Acc. Chem. Res. 2016, 49, 1389-1400. (b) Wang, J.; Dong, G. Palladium/Norbornene Cooperative Catalysis. Chem. Rev. 2019, 119, 7478-7528.

6. Shen, P.-X.; Wang, X.-C.; Wang, P.; Zhu, R.-Y.; Yu, J.-Q. Ligand-Enabled Meta-C-H Alkylation and Arylation using a Modified Norbornene. J. Am. Chem. Soc. 2015, 137, 11574-11577.

7. Wang, X.; Gensch, T.; Lerchen, A.; Daniliuc, C. G.; Glorius, F. Cp*Rh(III)/Bicyclic Olefin Cocatalyzed C-H Bond Amidation by Intramolecular Amide Transfer. J. Am. Chem. Soc. 2017, 139, 6506-6512.

8. Liu, J.; Ding, Q.; Fang, W.; Wu, W.; Zhang, Y.; Peng, Y. $\mathrm{Pd}(\mathrm{II}) /$ Norbornene-Catalyzed Meta-C-H Alkylation of Nosyl-Protected Phenylalanines. J. Org. Chem. 2018, 83, 13211-13216.

9. Wang, J.; Dong, Z.; Yang, C.; Dong, G. Modular and Regioselective Synthesis of All Carbon Tetrasubstituted Olefins Enabled by an Alkenyl Catellani Reaction. Nat. Chem. 2019, 11, 1106-1112.

10. Maestri, G.; Derat, E. Alkenyl boost for Catellani. Nat. Chem. 2019, 11, 1082-1084.

11. (a) Buchanan, G. L.; Bredt's Rule. Chem. Soc. Rev., 1974, 3, 41-63. (b) Elschenbroich, C. Organometallics. Weinheim: Wiley-VCH. 2006, ISBN 978-3-527-29390-2.

12. (a) Liu, G.; Shen, Y.; Zhou, Z.; Lu, X. Rhodium(III)Catalyzed Redox-Neutral Coupling of $N$-Phenoxyacetamides and Alkynes with Tunable Selectivity. Angew. Chem., Int. Ed. 2013, 52, 6033-6037. (b) Zhou, Z.; Bian, M.; Zhao, L.; Gao, H.; Huang, J.; Liu, X.; Yu, X.; Li, X.; Yi, W. 2HChromene-3- carboxylic Acid Synthesis via Solvent-Controlled and Rhodium(III)- Catalyzed Redox-Neutral C-H Activation/[3 + 3] Annulation Cascade. Org. Lett. 2018, 20, 3892-3896. (c) Zhong, X.; Lin, S.; Gao, H.; Liu, F.-X.; Zhou, Z.; Yi, W. Rh(III)-Catalyzed Redox-Neutral C-H Activation/[3 + 2] Annulation of N-Phenoxy Amides with Propargylic Monofluoroalkynes. Org. Lett. 2021, 23, 2285-2291.

13. (a) Gaussian 09, Revision A.03, M. J. Frisch, G. W. Trucks, H. B. Schlegel, G. E. Scuseria, M. A. Robb, J. R. Cheeseman, G. Scalmani, V. Barone, G. A. Petersson, H. Nakatsuji, X. Li, M. Caricato, A. Marenich, J. Bloino, B. G. Janesko, R. Gomperts, B. Mennucci. (b) Weigend, F.; Ahlrichs, R. Balanced Basis Sets of Split Valence, Triple Zeta Valence and Quadruple Zeta Valence Quality for H to Rn: Design and Assessment of Accuracy. Phys. Chem. Chem. Phys. 2005, 7, 3297-3305. (c) Weigend, F. Accurate Coulomb-Fitting Basis Sets for H to Rn. Phys. Chem. Chem. Phys. 2006, 8, 1057-1065. (d) Zhao, Y.; Truhlar, D. G. The M06 Suite of Density Functionals for Main Group Thermochemistry, Thermochemical Kinetics, Noncovalent Interactions, Excited States, and Transition Elements: Two New Functionals and Systematic Testing of Four M06-Class Functionals and 12 Other Function. Theor. Chem. Acc. 2008, 120, 215-241. (e) Marenich, A. V.; Cramer, C. J.; Truhlar, 
D. G. Universal Solvation Model Based on Solute Electron Density and on a Continuum Model of the Solvent Defined by the Bulk Dielectric Constant and Atomic Surface Tensions. J. Phys. Chem. B. 2009, 113, 6378-6396. (f) Grimme, S.; Antony, J.; Ehrlich, S.; Krieg, H. A Consistent and Accurate $\mathrm{Ab}$ Initio Parametrization of Density Functional Dispersion Correction (DFT-D) for the 94 Elements H-Pu. J. Chem. Phys. 2010, 132, 24103. (g) Grimme, S.; Ehrlich, S.; Goerigk, L. Effect of the Damping Function in Dispersion Corrected Density Functional Theory. J. Comput. Chem. 2011, 32, 1456-1465. (h) Guo, X. K.; Zhang, L. B.; Wei, D.; Niu, J. L. Mechanistic Insights into Cobalt(Ii/lii)-Catalyzed C-H Oxidation: A Combined Theoretical and Experimental Study. Chem. Sci. 2015, 6, 7059-7071. (i) Mardirossian, N.; Head-Gordon, M. How Accurate Are the Minnesota Density Functionals for Noncovalent Interactions, Isomerization Energies, Thermochemistry, and Barrier Heights Involving Molecules Composed of Main-Group Elements? J. Chem. Theory Comput. 2016, 12 (9), 4303-4325. (j) Li, X.; Wu, H.; Wu, Z.; Huang, G. Mechanism and Origins of Regioselectivity of Copper-Catalyzed Borocyanation of 2-Aryl-Substituted 1,3-Dienes: A Computational Study. J. Org. Chem. 2019, 84, 5514-5523. (k) Oliveira, J. C. A.; Dhawa, U.; Ackermann, L. Insights into the Mechanism of Low-Valent Cobalt-Catalyzed C-H Activation. ACS Catal. 2021, $11,1505-1515$.

14. (a) Gorelsky, S. I.; Lapointe, D.; Fagnou, K. Analysis of the Concerted Metalation-Deprotonation Mechanism in Palladium-Catalyzed Direct Arylation across a Broad Range of Aromatic Substrates. J. Am. Chem. Soc. 2008, 130, 10848-10849. (b) Lapointe, D.; Fagnou, K. Overview of the Mechanistic Work on the Concerted MetallationDeprotonation Pathway. Chem. Lett. 2010, 39, 11, 11181126. (c) R. Stuart, D.; Alsabeh, P.; Kuhn, M.; Fagnou, K. Rhodium(III)-Catalyzed Arene and Alkene C-H Bond Functionalization Leading to Indoles and Pyrroles. J. Am. Chem. Soc. 2010, 132, 18326-18339. (d) Ackermann, L. Carboxylate-Assisted Transition-Metal-Catalyzed C-H Bond Functionalizations: Mechanism and Scope. Chem. Rev. 2011, 111, 1315-1345. (e) Kapdi, A. R. Organometallic Aspects of Transition-Metal Catalysed Regioselective C-H Bond Functionalisation of Arenes and Heteroarenes. Dalt. Trans. 2014, 43, 3021-3034. (f) Davies, D. L.; Macgregor, S. A.; McMullin, C. L. Computational Studies of Carboxylate-Assisted C-H Activation and Functionalization at Group 8-10 Transition Metal Centers. Chem. Rev. 2017, 117, 86498709.

15. (a) Davies, D. L.; Donald, S. M. A.; Macgregor, S. A. Computational Study of the Mechanism of Cyclometalation by Palladium Acetate. J. Am. Chem. Soc. 2005, 127, 13754-13755. (b) Davies, D. L.; Donald, S. M. A.; Al-Duaij, O.; Macgregor, S. A.; Pölleth, M. Electrophilic $\mathrm{C}-\mathrm{H}$ Activation at $\{\mathrm{Cp} * \mathrm{Ir}\}$ : Ancillary-Ligand Control of the Mechanism of C-H Activation. J. Am. Chem. Soc. 2006, 128, 4210-4211. (c) Rousseaux, S.; Gorelsky, S. I.; Chung, B. K. W.; Fagnou, K. Investigation of the Mechanism of $\mathrm{C}(\mathrm{Sp} 3)-\mathrm{H}$ Bond Cleavage in $\mathrm{Pd}(0)$-Catalyzed
Intramolecular Alkane Arylation Adjacent to Amides and Sulfonamides. J. Am. Chem. Soc. 2010, 132, 1069210705. (d) Xiao, B.; Fu, Y.; Xu, J.; Gong, T.-J.; Dai, J.-J.; Yi, J.; Liu, L. Pd(II)-Catalyzed C-H Activation/Aryl-Aryl Coupling of Phenol Esters. J. Am. Chem. Soc. 2010, 132, 468-469.

16. (a) Wolinski, K.; Hinton, J. F.; Pulay, P. Efficient Implementation of the Gauge-Independent Atomic Orbital Method for NMR Chemical Shift Calculations. J. Am. Chem. Soc. 1990, 112,8251-8260. (b) Schleyer, P. V. R.; Maerker, C.; Dransfeld, A.; Jiao, H.; Van Eikema Hommes, N. J. R. Nucleus-Independent Chemical Shifts: A Simple and Efficient Aromaticity Probe. J. Am. Chem. Soc. 1996, 118, 6317-6318. (c) Merino, G.; Heine, T.; Seifert, G. The Induced Magnetic Field in Cyclic Molecules. Chem. - A Eur. J. 2004, 10, 4367-4371. (d) Chen, Z.; Wannere, C. S.; Corminboeuf, C.; Puchta, R.; von Ragué Schleyer, P. Nucleus-Independent Chemical Shifts (NICS) as an Aromaticity Criterion. Chem. Rev. 2005, 105, 3842-3888. (e) Ebrahimi, H. P.; Shaghaghi, H.; Tafazzoli, M. Gauge Invariant Atomic Orbital-Density Functional Theory Prediction of Accurate Gas Phase $1 \mathrm{H}$ and 13C NMR Chemical Shifts. Concepts Magn. Reson. Part A Bridg. Educ. Res. 2011, 38, 269-279.

17. (a) Stanger, A. Is the Mills Nixon Effect Real? J. Am. Chem. Soc. 1991, 113, 8277-8280. (b) Hodošček, M.; Kovaček, D.; Maksić, Z. B. Theoretical Study of Mills-Nixon Effect in Naphthocyclobutenes and -Cyclobutadienes. Theor. Chim. Acta 1993, 86, 343-351. (c) Siegel, J. S. MillsNixon Effect: Wherefore Art Thou? Angew. Chemie Int. Ed. English 1994, 33, 1721-1723. (d) Maksić, Z. B.; EckertMaksić, M.; Mó, 0.; Yáñez, M. The Mills-Nixon Effect: Fallacies, Facts and Chemical Relevance. In Pauling's Legacy; Maksić, Z. B., Orville-Thomas, W. J., Eds.; Theoretical and Computational Chemistry; Elsevier, 1999; Vol. 6, pp 47-101. (e) Eckert-Maksić, M.; Glasovac, Z.; Novak Coumbassa, N.; Maksić, Z. B. The Mills-Nixon Effect and Chemical Reactivity-Methyl Cation Affinity of Some Cycloalkabenzenes. J. Chem. Soc. Perkin Trans. 2 2001, No. 7, 1091-1098.

18. (a) Patel, P.; Chang, S. Cobalt(III)-Catalyzed C-H Amidation of Arenes using Acetoxycarbamates as Convenient Amino Sources under Mild Conditions. ACS Catal. 2015, 5, 853-858. (b) Lee, J.; Kang, B.; Kim, D.; Lee, J.; Chang. S.; Cobalt-Nitrenoid Insertion-Mediated Amidative Carbon Rearrangement via Alkyl-Walking on Arenes. J. Am. Chem. Soc. 2021, 143, 18406-18412.

19. (a) Tyman, J. H. P. Synthetic and Natural Phenols; Elsevier: New York, 1996. (b) Rappoport, Z. The Chemistry of Phenols; Wiley-VCH: Weinheim, 2003.

20. Sahoo, K.; Pradhan, P.; Panda, N. Access to C4-arylated benzoxazoles from 2-amidophenol through $\mathrm{C}-\mathrm{H}$ activation. Org. Biomol. Chem., 2020, 18, 1820-1832. 